\title{
The effect of head motion on the accuracy of sound localization
}

\author{
Masaharu Kato ${ }^{1,2, *}$, Hisashi Uematsu ${ }^{2, \dagger}$, Makio Kashino ${ }^{2}$ and Tatsuya Hirahara ${ }^{2}$ \\ ${ }^{1}$ Graduate School of Arts and Sciences, The University of Tokyo, \\ 3-8-1, Komaba, Meguro-ku, Tokyo, 153-8902 Japan \\ ${ }^{2}$ Human and Information Science Laboratory, NTT Communication Science Laboratories, NTT Corporation, \\ 3-1, Morinosato Wakamiya, Atsugi, 243-0198 Japan
}

( Received 8 January 2003, Accepted for publication 28 January 2003 )

Keywords: Sound localization, Head motion, HRTFs, Individual differences PACS number: 43.66.Qp, 43.66.Pn [DOI: 10.1250/ast.24.315]

\section{Introduction}

One of the crucial issues in virtual sound source localization using headphones is how to overcome the individual differences in head-related transfer functions (HRTFs). That is, when listening through HRTFs different from his/her own, a listener often complains that auditory events are spatially diffuse, and makes incorrect judgements about the source locations. Some methods have been proposed for resolving this $[1,2]$, with the common precondition that the head be fixed. In everyday life, however, most listeners are free to move around in the environment while listening to auditory events. Actually, the change in the binaural cue produced by head motion is effective in sound localization [3], and it is probably accurate to assume that this information induced by head motion is also useful for overcoming individual differences in HRTFs. We report here that differences from one's own HRTFs can be overcome by head motion. The effect of head motion on sound localization was examined when HRTFs were distorted by occluding a subject's concha. The effect of the direction of head motion was also examined by comparison with free, left-right only, up-down only, and no, head motion.

\section{Methods}

2.1. Apparatus and stimuli

Stimuli (white noise) were digitally synthesized on a computer with a sampling rate of $48 \mathrm{kHz}$ and quantization of 16 bits. The signals were then converted from digital to analog by a sound card (Digital Audio Labs. V8), and lowpass filtered (cut-off frequency $=20 \mathrm{kHz}$ ). Raised-cosine ramps of $100 \mathrm{~ms}$ duration were applied to the onset and offset of each stimulus. The duration of each stimulus was $10 \mathrm{~s}$, including the $100 \mathrm{~ms}$ ramps. The sound pressure level (SPL) at the center location of where the subject's head would be in the presence of the subject was adjusted to $57 \mathrm{~dB}$ SPL. The stimulus was freshly made before each presentation. A loudspeaker (JBL control MICRO) for delivering the stimulus was attached to an arc with a radius of $1,250 \mathrm{~mm}$. The end of the arc directly above the subject's head was attached to a stepping motor, so that it rotated around the vertical axis passing the center of the head, and that the center of the arc coincided with the center of the head. Another stepping motor

*Present Address: Tokyo Women's Medical University

${ }^{\dagger}$ Present Address: NTT Cyber Space Laboratories, NTT Corporation installed between the arc and the speaker moved the speaker up and down along the arc.

2.2. Subjects

Six females and one male aged between 22 and 29 years participated in the experiments. Their median age was 27 . The subjects were paid for their participation, and all had a normal hearing range of $500 \mathrm{~Hz}$ to $8 \mathrm{kHz}$.

\subsection{Design}

We used four conditions of head motion referred to as stationary, free, left-right and up-down. In stationary listening, each subject was instructed to keep his/her head facing forward and stationary throughout the block of trials. While in free motion listening, the subject was encouraged to move his/her head freely but not to move other parts of the body. In either left-right or up-down listening, the subject was encouraged to rotate his/her head horizontally or vertically without moving any other part of his/her body. Under these listening conditions, a gyration sensor (Polhemus Fastrack II) monitored the head motion for a period between $1 \mathrm{~s}$ before and $1 \mathrm{~s}$ after the stimulus, and a new block was restarted when any undesired motion was detected. There were also two conditions used to examine the importance of the spectral cue. In open-ear condition, the shape of the pinna was intact, whereas in ear mold condition, the foldings of the concha were filled with a thermoplastic ear mold, leaving the entrance of the external ear intact. These conditions were conducted in stationary and free listenings, thus the six conditions described in Table 1 were conducted with horizontal and vertical plane localizations. The order of these conditions was randomized.

\subsection{Procedure}

The experiments were conducted in a completely darkened anechoic room. Each subject wore a head-mounted display (Sony PLM-S700), through which instructions were given, and also a cap with the magnetic gyration sensor on top for monitoring the listener's head motion. The subject sat in a chair in the anechoic room, and the subject's task was to indicate the perceived sound source location after each stimulus presentation by pointing to the corresponding position on a schematic bird's-eye view (for horizontal localization) or side view (for vertical localization) displayed on the head-mounted display. No feedback on correct speaker position was given.

For horizontal localization, we divided $360^{\circ}$ of azimuth 
Table 1 Experimental conditions of ear molds and head movements. The numbers are corresponding to those in Figs. 1 and 2 .

\begin{tabular}{ccccc}
\hline & \multicolumn{4}{c}{ Head movements } \\
\cline { 2 - 5 } Ear molds & Stationary & Free & Left-right & Up-down \\
\hline NO & $(1)$ & $(2)$ & - & - \\
YES & $(3)$ & $(4)$ & $(5)$ & $(6)$ \\
\hline
\end{tabular}

into 12 sections in equal steps on the horizontal plane. One block of trials consisted of 12 stimulus presentations representing each of the 12 sections, and the order of the sections was randomized within a block. In each trial representing one section, speaker location was selected randomly with uniform probability within the section. The selected location was rounded to the nearest degree. A similar design was used for vertical localization, except that we divided the elevation range between $-42^{\circ}$ and $222^{\circ}$ into 11 sections. Accordingly, there were 11 stimulus presentations included in one block of trials.

In order to keep subjects from estimating the speaker's position based on the interval taken for it to move from one location to another, the between-trial interval was fixed at the time needed for the speaker to complete the longest path. Thus, for horizontal localization, the between-trial interval was $15 \mathrm{~s}$, while it was $55 \mathrm{~s}$ for vertical localization. It took 12 minutes to complete one block for horizontal localization and 20 minutes for vertical localization. As the stepping motors generated an audible noise, which could give the subjects an undesired localization cue, the subjects were asked to wear headphones (Sennheiser HDA-200), from which a dichotic sound was presented to mask the motor noise. The masking sound was white noise with no interaural correlation with $51 \mathrm{~dB}$ SPL. After an interval between trials, a chime was presented via the headphones and the subject then removed the headphones and pressed a button. The next stimulus was presented about $1 \mathrm{~s}$ after that.

\section{Results and discussion}

Figures 1 and 2 show the perceived location versus actual target locations. For the ease of reading, target and response locations were divided equally into blocks of ten degrees each. The numbers in the figures represent listening conditions in Table 1. Since there was no systematic difference between subjects in general, the results were pooled across all the subjects. The area of each circle is proportional to the response probability. The degree increased from lower front to lower back via above the subject in vertical localization (Fig. 1) and increased clockwise in horizontal localization (Fig. 2). To indicate localization accuracy, we computed rootmean-squared (RMS) errors for each condition. First, an error was computed by subtracting the response angle from the target. Then, for each subject and condition, the root mean square of errors across targets location was computed.

In general, in vertical localization, head motion improved localization accuracy; for example, comparing stationary to free listening (Fig. 1-1, Fig. 1-2), the average of individual RMS errors for all subjects decreased from $27^{\circ}$ to $13^{\circ}$. This decrease was also observed in a previous work [4,5].

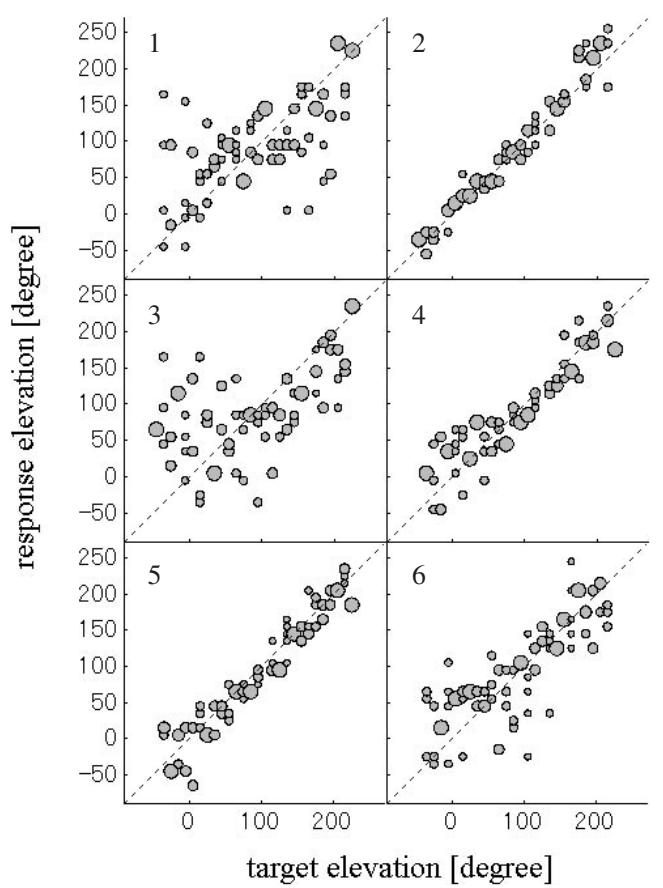

Fig. 1 Plots of target angle versus response for vertical localization. For details, see text.

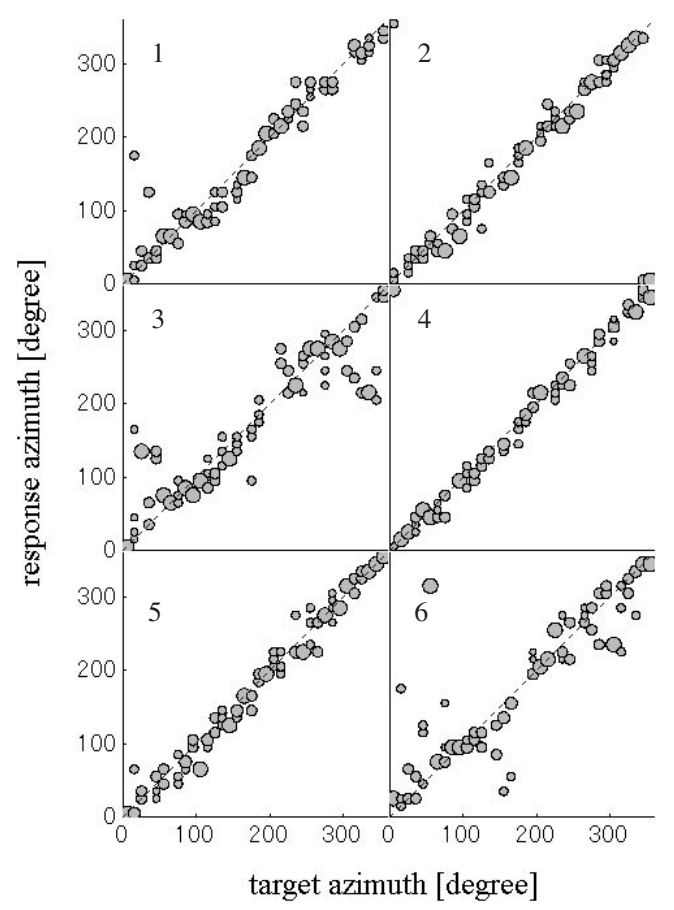

Fig. 2 Plots of target angle versus response for horizontal localization. For details, see text.

Furthermore, in horizontal localization, head motion with ear molds also contributed to the improvement of localization accuracy (Fig. 2-3, 2-4). However, no improvement was observed unless ear molds were used (Fig. 2-1, Fig. 2-2). This may be the ceiling effect, that is, the accuracy is high enough even without head motion. Comparing the distribution of data in Fig. 1 to that in Fig. 2, it was clear that the localization 
accuracy improved by head motion in vertical localization (Fig. 1), while in horizontal localization (Fig. 2), the decrease in front-back confusion contributed to the localization accuracy. Indeed, the significant difference between the accuracy of the stationary and the head-motion conditions (paired $t$-test with Holm correction, $p<0.05$ ) disappeared when we ignored the front-back dimension and used only the left-right dimension. Independent of the sound source direction, the accuracy in free motion and left-right motion conditions with ear molds was significantly better than in the stationary condition (paired $t$-test with Holm correction, $p<0.05$ ), which was almost the same as in free motion without ear molds. However, there was no significant difference between the up-down motion condition and the stationary condition. This means that the localization accuracy changes depending on the type of head motion.

\section{Conclusion}

In this study, we demonstrated that head motion overcame the difference from the listener's own HRTFs, which indicates that indivisual differences in HRTFs can be overcome by head motion. The up-down head motion, however, did not remove the decrease in localization accuracy. These results support that the changes in binaural cues make a major contribution to the localization accuracy.

\section{Acknowledgements}

We are grateful to Dr. Shigeto Furukawa for his helpful suggestions and discussions.

\section{References}

[1] J. C. Middlebrooks, "Virtual localization improved by scaling nonindividualized external-ear transfer functions in frequency," J. Acoust. Soc. Am., 106, 1493-1510 (1999).

[2] T. Inada, S. Yano, H. Hokari and S. Shimada, "A study on the individual deviation between ear canal transfer function and the inverse transfer function," Proc. Spring Meet. Acoust. Soc. Jpn., 3-P-6, pp. 531-532 (2000).

[3] H. Wallach, "The role of head movements and vestibular and visual cues in sound localization," J. Exp. Psychol., 27, 339368 (1940).

[4] S. Perrett and W. Noble, "The effect of head rotations on vertical plane sound localization," J. Acoust. Soc. Am., 102, 2325-2332 (1997).

[5] F. L. Wightman and D. J. Kistler, "Resolution of front-back ambiguity in spatial hearing by listener and source movement," J. Acoust. Soc. Am., 105, 2841-2853 (1999). 